\title{
Trans-thoracic Echocardiography in Prone Positioning COVID-19 Patients: a Small Case Series
}

\author{
Enrico Giustiniano ${ }^{1}$ (D) $\cdot$ Fabio Fazzari ${ }^{2} \cdot$ Renato Maria Bragato $^{2} \cdot$ Mirko Curzi $^{2} \cdot$ Maurizio Cecconi $^{1,3}$
}

Accepted: 9 September 2020 / Published online: 15 September 2020

(C) Springer Nature Switzerland AG 2020

\begin{abstract}
During SARS-CoV-2 pandemic, several subjects were treated in our intensive care unit (ICU) because of acute respiratory failure following COVID-19 pneumonia. Most of them required mechanical ventilation and someone in prone position (PP) too, because of acute respiratory distress syndrome (ARDS). During PP, trans-esophageal echocardiography (TEE) is not always easy, mainly due to the forced position of the neck of the patient. Moreover, during a pandemic, given the great number of patients needing treatment, TEE probes and monitoring devices are not widely available. Then, trans-thoracic echocardiography (TTE) plays a crucial role as it is non-invasive, repeatable, and available every time it is needed. Moreover, it can be safely performed also in prone position (TTEp). According to in-hospital protocol, TTEp was performed using the apical-four-chamber (A-4-C) view in 8 patients. We temporarily deflated the lower thoracic section of the air-mattress to place the probe between the mattress surface and the thorax of the patient. We collected both TEE and hemodynamics data. The main result of our retrospective analysis is that TTE can be performed in patients in prone positioning and is reliable and repeatable; the single apicalfour-chamber view provides sufficient data to evaluate the cardiac performance in case of scarce availability of hemodynamic monitoring devices, like in a pandemic setting. TTE may be a helpful tool for cardiac performance evaluation and diagnosis not only in supine or anterolateral positioning like in echocardiographic lab, but also in subjects admitted to ICU due to ARDS needing of mechanical ventilation in prone positioning.
\end{abstract}

Keywords Respiratory failure $\cdot$ Echocardiography $\cdot$ Coronavirus

\section{Introduction}

During SARS-CoV-2 pandemic, several subjects were treated in our intensive care unit (ICU) because of acute respiratory failure following COVID-19 pneumonia. Most of them required mechanical ventilation (MV) and some of them in prone position (PP) too, because of acute respiratory distress syndrome (ARDS), trying to re-open the collapsed and wet

This article is part of the Topical Collection on COVID-19

Enrico Giustiniano

enrico.giustiniano@humanitas.it

1 Department of Anesthesia and Intensive Care Units, Humanitas Clinical and Research Center, IRCCS, via Manzoni 56, Rozzano, 20089 Milan, Italy

2 Echocardiography Unit, Humanitas Clinical and Research Center, IRCCS, via Manzoni 56, 20089 Milan, Italy

3 Department of Biomedical Science, Humanitas University, via Rita Levi Montalcini 4, Pieve Emanuele, 20090 Milan, Italy lung parenchymal districts, particularly when severe hypoxemia occurred [1]. After a brief period of non-invasive ventilation (NIV), mechanical respiratory support was needed, since patients showed type $\mathrm{L}$ and/or type $\mathrm{H}$ of respiratory involvement. If in type L, clinical situation NIV may be the initial attempt to support the respiratory function, the type $\mathrm{H}$ clinical scenario makes MV mandatory since higher positive end-expiratory pressure (PEEP) levels and prone positioning are required, like in any type of ARDS [2-4].

During ARDS treatment, sedation and myorelaxation are required to make the patient be safely and efficaciously ventilated. Consequently, vasopressor administration may be necessary for hemodynamic support, both to counteract the vasodilation due to sedation and to provide a sufficient blood pressure, particularly when patient lays in prone position $[5,6]$. Such a critical illness demands a strict cardiovascular monitoring and repeated cardiac function assessment. Hemodynamic monitoring can be performed by right heart catheterization, trans-pulmonary thermodilution (TPT) technique, or echocardiography. The latest offers the advantage to evaluate both cardiac function and hemodynamic status 
non-invasively and in a cost-saving way $[6,7]$. Peng et al. reported that during the COVID-19 pandemic in China, the main cardiocirculatory abnormal findings were as follows: (1) hyperdynamic state; (2) stress-induced cardiomyopathy (Takotsubo); (3) right ventricle enlargement and acute pulmonary hypertension; (4) diffuse myocardial inhibition [8].

During prone positioning, trans-esophageal echocardiography (TEE) is not always easy, mainly due to the forced position of the neck of the patient. Moreover, during a pandemic, given the great number of patients needing treatment, TEE probes and monitoring devices are not widely available. Then, trans-thoracic echocardiography (TTE) plays a crucial role as it is non-invasive, repeatable, and available for many patients every time it is needed; moreover, it can be safely performed also in prone position (TTEp), as we described in a previous report [9].

In this small case series, we report our experience about TTEp in mechanically ventilated patients admitted to COVID19 isolation intensive care unit (ICU-COVID-19).

\section{Methods}

\section{Clinical Data and Patients' Selection}

We retrospectively analyzed electronic medical records of 107 patients admitted to our ICU-COVID-19 from March 1 to May 21, 2020, during the new coronavirus 2019 outbreak in Italy. Only 8 of them received echocardiographic evaluation both in supine and in prone positions and were included in the study. Patients' clinical data are summarized in Table 1. Clinical variables were as follows: age, sex, body mass index, comorbidities, mean arterial pressure (MAP), heart rate (HR), mechanical ventilation measurements like PEEP, and respiratory system compliance $\left(C_{\mathrm{rs}}\right)$. Only few patients included into the study received trans-pulmonary thermo-dilution hemodynamic monitoring. None of the included cases received pulmonary artery catheter (PAC) hemodynamic monitoring.

According to our institutional protocol, all patients received non-invasive ventilation (NIV) as first choice. When respiratory function deteriorated despite NIV, patients have been intubated and admitted to our ICU-COVID-19 to receive invasive mechanical ventilation. In case of severe hypoxemia $\left(\mathrm{pO}_{2} / \mathrm{FiO}_{2}<150 \mathrm{mmHg}\right)$ despite PEEP $\geq 10 \mathrm{cmH}_{2} \mathrm{O}$, they received mechanical ventilation in prone positioning (PPMV). Before declaring the patient unresponsive to PP$\mathrm{MV}$ (i.e., $\mathrm{pO}_{2} / \mathrm{FiO}_{2}<150 \mathrm{mmHg}$ with $\mathrm{PEEP} \geq$ $10 \mathrm{cmH}_{2} \mathrm{O}$ ), the PP cycle was attempted for three times. Each PP cycle included $16-18 \mathrm{~h}$ in prone positioning followed by $6-8 \mathrm{~h}$ of supine positioning (SP).
Table 1 Results

\begin{tabular}{|c|c|c|c|}
\hline \multirow[b]{2}{*}{ Age (years) } & \multicolumn{3}{|c|}{ COVID-19 patients $(n=8)$} \\
\hline & $61.7 \pm 7.9$ & & \\
\hline Gender (male/female) & $7 / 1$ & & \\
\hline \multirow[t]{2}{*}{$\operatorname{BSA}\left(\mathrm{m}^{2}\right)$} & $2.1 \pm 0.2$ & & \\
\hline & Supine ${ }^{(1)}$ & Prone $^{(2)}$ & $p$ \\
\hline \multicolumn{4}{|l|}{ Hemodynamics } \\
\hline MAP (mmHg) & $83 \pm 12$ & $78 \pm 14$ & 0.417 \\
\hline HR (bpm) & $100 \pm 28$ & $100 \pm 11$ & 1.000 \\
\hline $\mathrm{ScvO}_{2}(\%)$ & $74 \pm 4.8$ & $77 \pm 5.2$ & 0.270 \\
\hline CVP (mmHg) & $11 \pm 2.1$ & N/A & - \\
\hline Norepinephrine ( $\mu \mathrm{g} / \mathrm{kg} / \mathrm{min})$ & $0.17 \pm 0.1$ & $0.22 \pm 0.2$ & 0.500 \\
\hline \multicolumn{4}{|l|}{ Mechanical ventilation } \\
\hline $\mathrm{pO}_{2} / \mathrm{FiO}_{2}(\mathrm{mmHg})$ & $110 \pm 19$ & $121 \pm 32$ & 0.456 \\
\hline PEEP $\left(\mathrm{cmH}_{2} \mathrm{O}\right)$ & $13 \pm 3.0$ & $15 \pm 2.6$ & 0.265 \\
\hline$C_{\mathrm{rs}}(\mathrm{ml} / \mathrm{cmH} 2 \mathrm{O})$ & $34 \pm 9.2$ & $35 \pm 11.7$ & 0.780 \\
\hline \multicolumn{4}{|l|}{ Echocardiography } \\
\hline $\mathrm{RV}$ end-diastolic diameter (mm) & $37 \pm 5$ & $35 \pm 3$ & 0.328 \\
\hline LV end-diastolic diameter (mm) & $43 \pm 4$ & $44 \pm 5$ & 0.704 \\
\hline $\mathrm{RV} / \mathrm{LV}$ end-diastolic diameter ratio & $0.88 \pm 0.1$ & $0.81 \pm 0.1$ & 0.348 \\
\hline TAPSE (mm) & $21 \pm 3$ & $21 \pm 5$ & 0.954 \\
\hline $\mathrm{S}^{\prime}$ TDI tricuspid valve annulus $(\mathrm{cm} / \mathrm{s})$ & $16 \pm 3.8$ & $19 \pm 9.3$ & 0.398 \\
\hline PAPs (mmHg) & $49 \pm 9$ & $41 \pm 10$ & 0.132 \\
\hline LVOT VTI (cm) & $20.6 \pm 4.9$ & $25.6 \pm 5.8$ & 0.184 \\
\hline LV ejection fraction $(\%)$ & $53 \pm 4.6$ & $54 \pm 5.8$ & 0.815 \\
\hline MAPSE (mm) & $13 \pm 1.9$ & $16 \pm 4.9$ & 0.171 \\
\hline IVC maximum diameter & $21 \pm 2.9$ & $-*$ & \\
\hline IVC distensibility index (\%) & $25 \pm 21$ & $-*$ & \\
\hline
\end{tabular}

$M A P$, mean arterial pressure; $\mathrm{HR}$, heart rate; $\mathrm{ScvO}_{2}$, central venous oxygen saturation; $C V P$, central venous pressure; $P E E P$, positive endexpiratory pressure; $C_{r s}$, compliance of the respiratory system; $R V$, right ventricle; $L V$, left ventricle; TAPSE, tricuspid annular plane systolic excursion; $S^{\prime} T D I$, systolic wave tissue Doppler imaging; PAPS, pulmonary artery systolic pressure; LVOT VTI, left ventricle outflow tract velocitytime integral; MAPSE, mitralic annular plane systolic excursion; IVC, inferior vena cava; $N / A$, not available

${ }^{(1)}$ Supine position: just before the prone positioning

${ }^{(2)}$ Prone position: $\geq 1 \mathrm{~h}$ after the prone positioning

*In prone position, the subcostal view is not accessible

Protective ventilation protocol included tidal volume $(\mathrm{Vt})$ of 4-6 $\mathrm{ml} / \mathrm{kg}$ and PEEP $\leq 20 \mathrm{cmH}_{2} \mathrm{O}$ with target driving pressure (DP) $\leq 15 \mathrm{cmH}_{2} \mathrm{O}$ and airway plateau pressure $\left(P_{\text {plat }}\right) \leq$ $35 \mathrm{cmH}_{2} \mathrm{O}$. The compliance of the respiratory system $\left(C_{\mathrm{rs}}\right)$ if not provided by the ventilator software was computed by the following formula: Vt/DP $\left(\mathrm{ml} / \mathrm{cmH}_{2} \mathrm{O}\right)$, where DP results from the difference $\left(P_{\text {plat }}-\right.$ PEEP).

All the patients underwent thoracic $\mathrm{CT}$ scan before prone positioning when it was possible according to the logistic availability and the patient safety. 
The evolution from interstitial pneumonia to ARDS was diagnosed according the Berlin Definition [10].

All the patients received invasive blood pressure monitoring by an arterial line.

The first-choice drug to support hemodynamics was norepinephrine (NE), followed by dobutamine (DB) when inotropic support was needed, targeting a cardiac index $>21 / \mathrm{kg} /$ min (measured by TPT of TTE) and a mean arterial pressure $(\mathrm{MAP}) \geq 65 \mathrm{mmHg}$.

\section{Echocardiographic Data}

Trans-thoracic echocardiography was performed as stated by guidelines using the US machine GE-Venue (CGeneral Electric Healthcare, Little Chalfont, Buckinghamshire, UK).

According to in-hospital protocol, TTEp was performed using the apical-four-chamber (A-4-C) view. We temporarily deflated the lower thoracic section of the air-mattress to place the probe between the mattress surface and the thorax of the patient (Fig. 1).

The following echocardiographic data were collected: LV ejection fraction (LVEF); the mitralic annular plane excursion (MAPSE); the left ventricle outflow tract velocity-time integral (LVOT-VTI); the ratio between the RV end-diastolic diameter and LV end-diastolic diameter (RV/LV) measured at the mid-basal level in the A-4-C view; the RV systolic performance by the tricuspid annular plane excursion (TAPSE); tricuspid regurgitation pressure gradient to estimate the pulmonary artery systolic pressure (PAPs).

We assumed as normal RV-EDD $\leq 34 \mathrm{~mm}, \mathrm{LV}-\mathrm{EDD} \leq$ $53 \mathrm{~mm}$, and normal ratio RV/LV EDD $\leq 0.5$, [11-13].

Echocardiographic examinations in prone and supine positions were performed by one experienced operator, and echocardiographic recordings were post hoc analyzed by two independent readers (one anesthesiologist and one cardiologist) (Fig. 2).

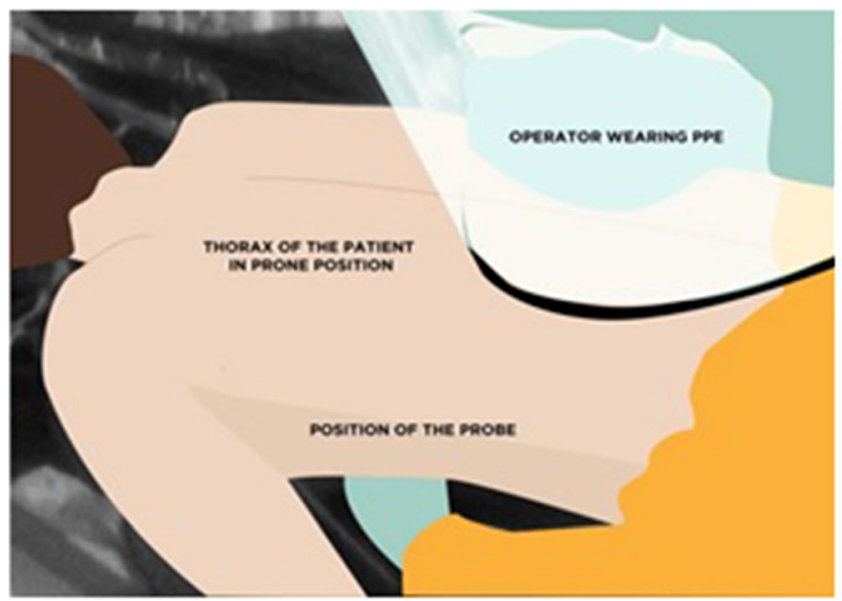

Fig. 1 TTE $_{\mathrm{p}}$ technique and the A-4-C view
When the patient was considered for prone positioning, he/ she received a supine TTE. The TTEp was performed within 30-60 min after prone positioning.

The Independent Ethics Committee of Humanitas Clinical and Research Center (IRB approval n. 233/20, on March 12th 2020) approved the study and given its retrospective and observational design waived the need for individual informed consent.

We followed the STROBE Statement for observational study on human subjects (http://www.strobe-statement.org/).

\section{Statistics}

Results are reported as average \pm standard deviation (SD) or median and range enclosed in parentheses, or number and percentage enclosed in parentheses, as appropriate. Percentage variation between SP and PP data was computed with the following formula:

[(Prone value-Supine value)/Supine value]

We adopted Student's $t$ test for parametric data analysis while for non-parametric variables, we adopted Fisher's exact test. Level of statistical significance was $p<0.05$.

Analysis was performed with the Prism 8.2.1 software (GraphPad, 2356 Northside Dr., Suite 560, San Diego, CA 92108, USA).

\section{Results}

Among the 8 patients included in our analysis, none of them needed dobutamine administration. Conversely, all of them received norepinephrine to sustain hemodynamics and reach a $\mathrm{MAP} \geq 65 \mathrm{mmHg}$.

Table 1 reports the main results.

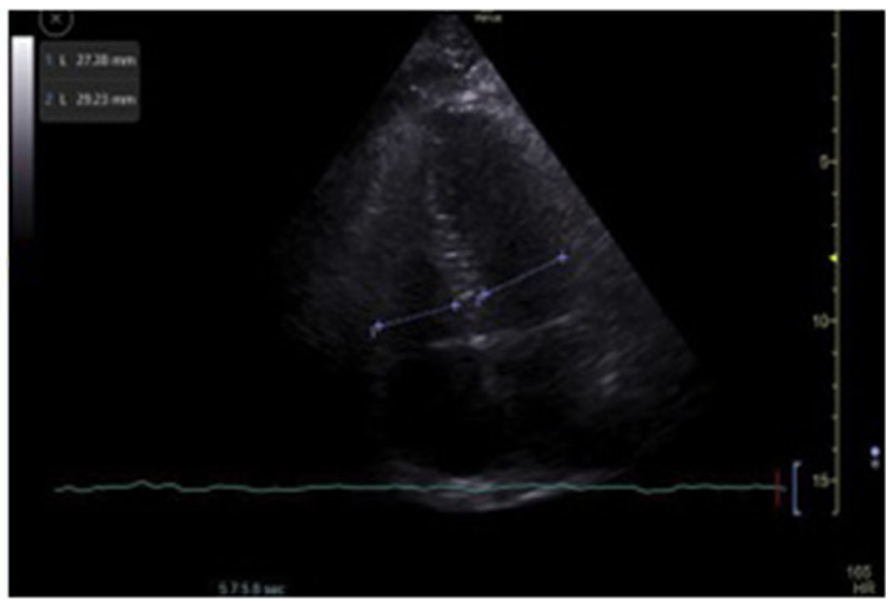


a

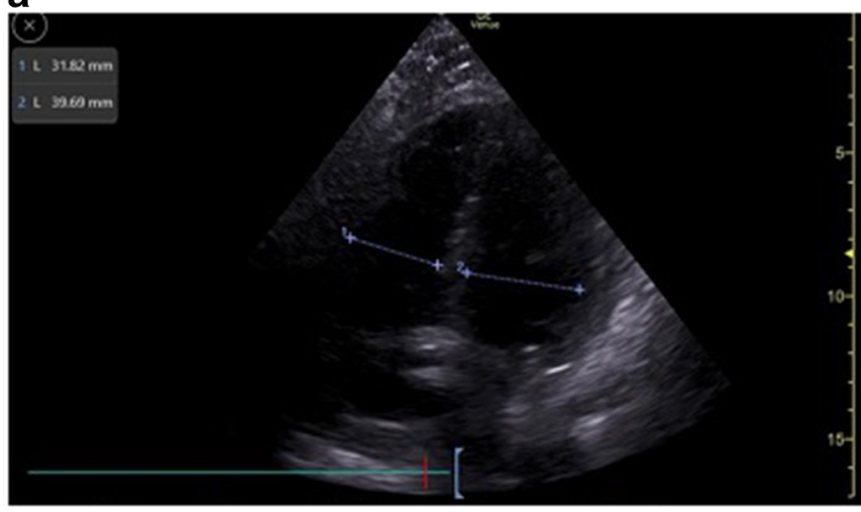

b

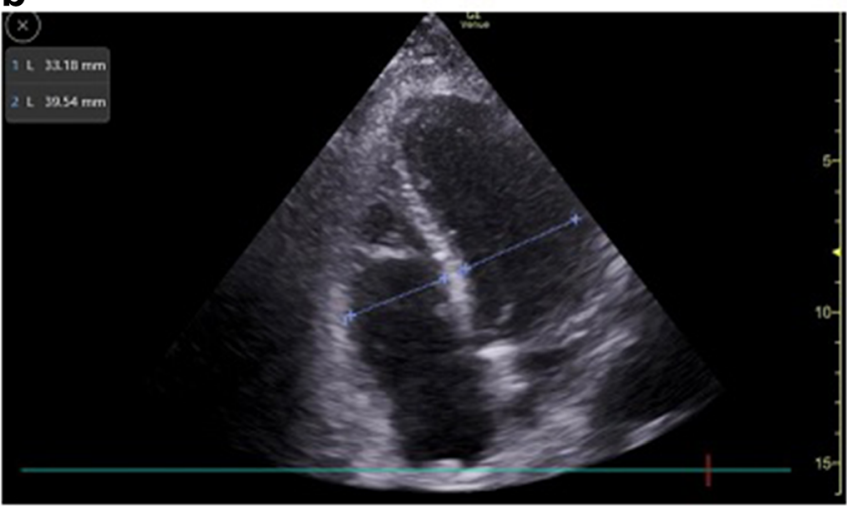

Fig. 2 One case COVID-19 example of diameter ventricle ratio in supine and prone positioning echocardiography. a Echocardiography in supine positioning. b Echocardiography in prone positioning

\section{Baseline Echocardiographic Findings in Supine Position}

LVEF was normal in $87 \%$ of patients (7/8 had a LVEF more than 50\%), and right ventricular function expressed as TAPSE was under the lower reference limit in one patient (12\% of cases, mean value 21). RV dimension was over the upper limit in all patients with a mean value of RV/LV ratio of 0.87 .

Eighty-seven percent of patients showed high PAPs (mean value of 49) and inferior vena cava was dilated in 5 patients (62\%); only one patient showed a caval index more than $50 \%$.

\section{Hemodynamic and Respiratory Changes in Prone Position}

MAP showed a little but not significant lowering. At the same time, the other hemodynamic parameters did not change significantly.

Respiratory data showed not any modification, even the $\mathrm{pO}_{2} / \mathrm{FiO}_{2}$ ratio which was expected to improve significantly.

\section{Echocardiographic Changes in Prone Position}

As showed in Table 1, the majority of patients $(6 / 8,75 \%)$ showed no change in LVEF, while the remaining two patients showed respectively improvement and worsening of LVEF.

$\mathrm{RV}$ diameter reduced in $5 / 8$ patients $(62.5 \%)$ and increased in 2 patients. PAPs lowered in $6 / 8$ patients and augmented only in one patient.

\section{Case-by-Case Analysis}

The greatest negative variation of TAPSE observed was in case n. $2(-43 \%)$ with a worsening of PAPs too $(+9 \%)$, a little reduction of $P / F(-2 \%)$, and a great variation of respiratory compliance $\left(C_{\mathrm{rs}}-26 \%\right)$ (Table 2$)$. This patient was the only one who died after the shortest ICU staying (12 days).

\section{Outcomes}

Mortality rate of this small group of patients was $12.5 \%$ (only one patient died). Among survivors, the mean
Table 2 Patients' data after prone positioning and outcome

\begin{tabular}{llllllll}
\hline Patient & $\begin{array}{l}\text { TAPSE } \\
\text { variation } \\
(\%)\end{array}$ & $\begin{array}{l}\text { RVEDD } \\
\text { variation } \\
(\%)\end{array}$ & $\begin{array}{l}P / F \\
\text { variation } \\
(\%)\end{array}$ & $\begin{array}{l}C_{\mathrm{rs}} \\
\text { variation } \\
(\%)\end{array}$ & $\begin{array}{l}\text { PAPs } \\
\text { variation } \\
(\%)\end{array}$ & $\begin{array}{l}\text { ICU length } \\
\text { of stay } \\
(\text { days })\end{array}$ & Outcome \\
\hline 1 & -5 & -26 & 27 & 39 & -17 & 90 & Alive \\
2 & -43 & -3 & -2 & -26 & 9 & 12 & Dead \\
3 & 53 & -15 & 30 & -26 & -42 & 64 & Alive \\
4 & 16 & -3 & 22 & 22 & -19 & 35 & Alive \\
5 & -22 & 3 & 9 & 68 & -17 & 64 & Alive \\
6 & -9 & 0 & 7 & 14 & -17 & 49 & Alive \\
7 & -13 & 9 & 4 & -20 & -23 & 24 & Alive \\
8 & 42 & -3 & -24 & 0 & 0 & 66 & Alive \\
\hline
\end{tabular}

TAPSE, tricuspid annular plane systolic excursion; $R V E D D$, right ventricle end-diastolic diameter; $P / F, \mathrm{pO}_{2} / \mathrm{FiO}_{2}$ ratio; $C_{r s}$, compliance of the respiratory system; $P A P S$, systolic pulmonary artery pressure 
length of stay was 56 days, with 5 patients having an unfavorable long hospitalization of more than 31 days.

\section{Discussion}

The main results of our retrospective analysis on a small cases series consisted of few main points: (1) TTE can be performed in patients in prone positioning and is reliable in comparison with the supine TTE and repeatable, meaning that it may be used also to "monitor" the cardiac function every time it is needed, in a standardized way (i.e., without any minimal changing of the patient position, during both supine and prone positioning); the single apical-four-chamber view provides sufficient data to evaluate the cardiac performance in case of scarce availability of hemodynamic monitoring devices, like in a pandemic setting; (2) according to recent scientific reports, prone positioning in patients suffering from ARDS due to COVID-19 pneumonia is not always effective. It may depend on the phenotype of the disease and may be effective only in extremely severe cases [1-4].

We observed a reduction of PAPs and RV-EDD after prone positioning. We may speculate that PAPs lowering may be the effect of a supposed reduction of pulmonary resistances after prone positioning. It may be the cause of the reduction of the RV-EDD. This may translate in an improved RV function as showed by the increase of $\mathrm{S}^{\prime}$ TDI tricuspid valve annulus (Table 1). Unfortunately, these variations did not reach a statistical significance, but it may be due to the small sample of the trial.

In our small case series, only one patient died. This patient showed a reduction of the RV and LV performances along with an increase in PAPs and a worsening of respiratory compliance in prone position. We may suppose that the reduction of pulmonary compliance caused the worsening of the RV function and increase of PAPs. The course of this case was also more rapid compared with the others.

The TTE in prone positioning $\left(\mathrm{TTE}_{\mathrm{p}}\right)$ was helpful to evaluate the hemodynamic state of our patients despite they did not receive a specific monitoring methods (trans-pulmonary thermodilution, pulmonary artery catheter, or semi-invasive pulse-contour systems). Despite its diagnostic primary property and because we had not a sufficient number of hemodynamic monitoring devices, $\mathrm{TTE}_{\mathrm{p}}$ resulted a valid alternative to classical hemodynamic monitoring. It is safe, repeatable, and cost saving.

While TEE performed in the prone positioning has been reported as a safe procedure [14], in our opinion, one limitation may be the position of the neck and head of the patient that is laterally rotated and may make it difficult to introduce the TEE probe. Additionally, given the potential high number of patients requiring this type of evaluation and the number of devices available, TEE is often a logistic challenge.
Our study has a lot of limitations. The main are as follows: (1) the sample is too small to permit definitive conclusions which in this case could be only speculative; (2) it is a retrospective analysis of data collected in a situation of extreme difficulty like during a pandemic; then, several further data that are helpful for a more detailed analysis are not available because our database is lacking.

In conclusion, TTE may be a helpful tool for cardiac performance evaluation and diagnosis not only in supine or anterolateral positioning like in echocardiographic lab, but also in subjects admitted to ICU due to ARDS needing of mechanical ventilation in prone positioning. In particular, our "trick" (i.e., mattress deflation) permits to perform TTE in prone positioning without any minimal changing of the patient position.

\section{Compliance with Ethical Standards}

The study was approved by the Independent Ethical Committee of the Humanitas Clinical and Research Center on March 12th, 2020 (IRB n. 233/20)

Conflict of Interest The authors declare that they have no conflict of interest.

\section{References}

1. Taccone P, Pesenti A, Latini R, Polli F, Vagginelli F, Mietto C, et al. Prone positioning in patients with moderate and severe acute respiratory distress syndrome: a randomized controlled trial. JAMA. 2009;302(18):1977-84. https://doi.org/10.1001/jama. 2009.1614.

2. Marini JJ, Gattinoni L. Management of COVID-19 respiratory distress. JAMA. 2020;24:E1-2.

3. Gattinoni L, Coppola S, Cressoni M, Busana M, Rossi S, Chiumello D. Covid-19 does not lead to a "typical" acute respiratory distress syndrome. Am J Respir Crit Care Med. 2020. https:// doi.org/10.1164/rccm.202003-0817LE [published online ahead of print, 2020 Mar 30].

4. Gattinoni L, Chiumello D, Rossi S. COVID-19 pneumonia: ARDS or not? Crit Care. 2020;24:154.

5. Zochios V, Parhar K, Tunnicliffe W, Roscoe A, Gao F. The right ventricle in ARDS. Chest. 2017;152(1):181-93. https://doi.org/10. 1016/j.chest.2017.02.019.

6. Vieillard-Baron A, Girou E, Valente E, Brun-Buisson C, Jardin F, Lemaire $\mathrm{F}$, et al. Predictors of mortality in acute respiratory distress syndrome. Focus on the role of right heart catheterization. Am J Respir Crit Care Med. 2000;161(5):1597-601.

7. Mitchell C, Rahko PS, Blauwet LA, Canaday B, Finstuen JA, Foster MC, et al. Guidelines for performing a comprehensive transthoracic echocardiographic examination in adults: recommendations from the American Society of Echocardiography. J Am Soc Echocardiogr Jan. 2019;32(1):1-64. https://doi.org/10.1016/j.echo. 2018.06.004.

8. Peng QY, Wang XT, Zhang LN, Chinese Critical Care Ultrasound Study Group (CCUSG). Using echocardiography to guide the treatment of novel coronavirus pneumonia. Crit Care. 2020;24(1):143. Published 2020 Apr 10. https://doi. org/10.1186/s13054-020-02856-z. 
9. Giustiniano E, Bragato RM, Padua E, Negri K, Cecconi M. Echocardiography during prone-position mechanical ventilation in patients with COVID-19: a proposal for a new approach. Journal of American Society of Echocardiography. 2020. https:// doi.org/10.1016/j.echo.2020.04.027 [article in press].

10. Ashbaugh DG, Bigelow DB, Petty TL, Levine BE. Acute respiratory distress in adults. Lancet. 1967;2:319-23.

11. Lang RM, Bierig M, Devereux RB, Flachskampf FA, Foster E, Pellikka PA, et al. Recommendations for chamber quantification: a report from the American Society of Echocardiography's Guidelines and Standards Committee and the Chamber Quantification Writing Group, developed in conjunction with the European Association of Echocardiography, a branch of the European Society of Cardiology. J Am Soc Echocardiogr. 2005;18(12):1440-63.

12. Lang RM, Badano LP, Mor-Avi V, Afilalo J, Armstrong A, Ernande L, et al. Recommendations for cardiac chamber quantification by echocardiography in adults: an update from the American Society of Echocardiography and the European Association of Cardiovascular Imaging. J Am Soc Echocardiogr. 2015;28(1):1-39.e14. https://doi.org/10.1016/j.echo.2014.10.003.

13. Fan E, Brodie D, Slutsky AS. Acute Respiratory Distress Syndrome: Advances in Diagnosis and Treatment. JAMA. 2018;319(7):698-710. https://doi.org/10.1001/jama.2017.21907.

14. Mekontso Dessap A, Proost O, Boissier F, Louis B, Roche Campo F, Brochard L. Transesophageal echocardiography in prone position during severe acute respiratory distress syndrome. Intensive Care Med. 2011;37(3):430-4. https://doi.org/10.1007/s00134010-2114-z.

Publisher's Note Springer Nature remains neutral with regard to jurisdictional claims in published maps and institutional affiliations. 